\title{
TWISTED SUMS OF NUCLEAR FRÉCHET SPACES
}

\author{
TIMO KETONEN AND KAISA NYBERG
}

\section{Introduction}

In proving that a nuclear Fréchet space $F$ with the property $\mathrm{DN}$ is a subspace of $s$, Vogt [11] showed that there is a short exact sequence $0 \rightarrow s \rightarrow X \rightarrow F \rightarrow 0$, where $X$ is a subspace of $s$. Using the property DN he showed next that this sequence splits. It follows that $F$ is a subspace of $s$. This kind of technique has proved to be useful in characterization of subspaces and quotient spaces of nuclear Fréchet spaces, cf. [1], [2], [12], and [13]. We will take another point of view for investigating short exact sequences. Let $E$ and $F$ be nuclear Fréchet spaces. The problem is to construct a nontrivial twisted sum of the spaces $E$ and $F$, i.e., to construct a space $X$ such that it has a subspace $Y$ isomoprhic to $E$ with $X / Y$ isomorphic to $F$, and such that $Y$ is not complemented in $X$. In [9] Kalton and Peck gave a general method of constructing twisted sums of sequence spaces. This is our starting point. In Section 2 we show that also in the case of nuclear Fréchet spaces there is a general method of constructing twisted sums, provided that the quotient space has a basis. In Section 3 we construct nontrivial examples of short exact sequences of the type $0 \rightarrow \Lambda_{1}(\alpha) \rightarrow X \rightarrow \Lambda_{1}(\beta) \rightarrow 0$ and $0 \rightarrow \Lambda_{\infty}(\alpha) \rightarrow X \rightarrow \Lambda_{1}(\beta) \rightarrow 0$. We conclude in Section 4 by giving a fairly general splitting condition when both the subspace and the quotient space have bases.

For undefined terminology we refer to [4] and [10]. The scalar field is assumed to be the field of real numbers.

\section{Construction of twisted sums}

We recall that $X$ is a twisted sum of topological vector spaces $E$ and $F$ if there is a short exact sequence $0 \rightarrow E \rightarrow X \rightarrow F \rightarrow 0$, i.e., if $E$ is isomorphic to a subspace of $X$ so that the quotient space is isomorphic to $F$. In their work [9] Kalton and Peck constructed some very interesting examples of twisted sums of Banach spaces. They did this by constructing a quasilinear map $G: F \rightarrow E$, and then defining the space $X$ to be the space $E \times F$ with the quasinorm $|(x, y)|=\|G y-x\|+\|y\|$. In the case of Banach spaces there is the problem that a twisted sum need not be a Banach space, it is not necessarily locally convex [7]. On the other hand, Kalton [7] has shown that 
a twisted sum of for example Hilbert spaces is locally convex. This, and the fact that a nuclear Fréchet space is a projective limit of Hilbert spaces, will imply that local convexity is not a problem in the case of nuclear Fréchet spaces.

Lemma 2.1. (see [3, Hilfsatz 5.8]). Let $E$ and $F$ be topological vector spaces. Assume we have short exact sequences

and

$$
0 \rightarrow E \stackrel{j}{\rightarrow}\left(X, \tau_{1}\right) \stackrel{q}{\rightarrow} F \rightarrow 0
$$

$$
0 \rightarrow E \stackrel{\dot{j}}{\rightarrow}\left(X, \tau_{2}\right) \stackrel{q}{\rightarrow} F \rightarrow 0 .
$$

If $\tau_{1} \subset \tau_{2}$, then $\tau_{1}=\tau_{2}$.

Proof, For completeness we give the simple proof. Let $U$ be $\tau_{2}$-neighbourhood of the origin. Choose a $\tau_{1}$-neighbourhood $V$ of the origin in such a way that $(V-V) \cap$ $j(E) \subset U \cap j(E)$, and let $W \subset V$ be a $\tau_{1}$-neighbourhood of the origin such that $W \subset U \cap V+j(E)$. We have $W \subset U \cap V+j(E) \cap(W-U \cap V) \subset U+j(E) \cap(V-V) \subset$ $U+U$.

Theorem 2.2. A twisted sum of nuclear Fréchet spaces is a nuclear Fréchet space.

Proof. First of all, a twisted sum of complete metric linear spaces is a complete metric linear space. That it has a countable fundamental system of neighbourhoods of the origin is a consequence of the above lemma, whereas completeness can be verified directly. Secondly, Kalton [8] has shown that a twisted sum of the real line and a nuclear Fréchet space is locally convex, i.e., every short exact sequence $O \rightarrow \boldsymbol{R} \rightarrow$ $X \rightarrow F \rightarrow O$, where $F$ is a nuclear Fréchet space, splits. This implies that a twisted sum of nuclear Fréchet spaces is locally convex [3, Satz 2.4.1.]. Finally, to prove the nuclearity, it is not hard to verify that for every continuous seminorm there is a continuous seminorm such that summability with respect to the first one implies absolute summability with respect to the second one [3, Satz 2.3.5].

The above theorem gives us free hands for constructing twisted sums of nuclear Fréchet spaces; we always get a nuclear Fréchet space. In the case of Banach spaces every twisted sum can be expressed with the help of a quasilinear map [9, Theorem 2.4.]. However, in order to construct quasilinear maps one needs some simplifying assumptions, e.g. the existence of a suitable basis. On the other hand, if a nuclear Fréchet space has a basis, it is absolute [6]. This gives us an additional simplification in that we do not have to use quasilinear maps, but linear maps will suffice.

Let $E$ and $F$ be nuclear Fréchet spaces. Without loss of generality we may assume that their topologies are defined by an increasing sequence of seminorms $\left(\|\cdot\|_{p}\right)$ and $\left(|| \cdot|| \|_{p}\right)$, respectively, such that the corresponding unit balls form a neighbourhood basis of the origin. Let $F_{0}$ be a dense subspace of $F$. Assume we have a sequence of linear maps $A_{p}: F_{0} \rightarrow E$ which satisfy the following compatibility condition: 
There is a function $\sigma: N \rightarrow \boldsymbol{N}$ such that for every $p$ and $q, p>q$, we have

$$
\left\|A_{p} y-A_{q} y\right\|_{q} \leqq\|y\| \|_{\sigma(p)}, \quad y \in F_{0} .
$$

Note that we do not require continuity of the linear maps $A_{p}$. Note also that the function $\sigma$ can be chosen to be strictly increasing. Define $E \times_{\left(A_{p}\right)} F_{0}$ to be the space $E \times F_{0}$ equipped with the topology given by the seminorms

$$
|(x, y)|_{p}=\left\|A_{p} y-x\right\|_{p}+\left\||| y|\||_{\sigma(p)}, \quad p \in \mathbf{N} .\right.
$$

Here we could have used any sequence of seminorms

$$
|(x, y)|_{n}=C_{n}\left\|A_{p_{n}} y-x\right\|_{p_{n}}+D_{n}\left|\left\|y|\||_{q_{n}},\right.\right.
$$

where $C_{n}>0, D_{n}>0$, and $\lim _{n \rightarrow \infty} p_{n}=\lim _{n \rightarrow \infty} q_{n}=\infty$; the topology does not change as we see by using the compatibility assumption. The reason for our choice is that in this way, increasing the values of the function $\sigma$ appropriately, we get an increasing sequence of seminorms.

Lemma 2.3. The sequence $0 \rightarrow E \stackrel{j}{\rightarrow} E \times_{\left(A_{p}\right)} F_{0} \underline{q_{0}} \rightarrow F_{0} \rightarrow 0$, where $j(x)=(x, 0)$ and $q_{0}(x, y)=y$, is exact.

Proof. Since $|j(x)|_{p}=|(x, 0)|_{p}=\|x\|_{p}, j$ is an isomorphism onto its image. As regards the quotient map $q_{0}$, we have

$$
\begin{gathered}
\left\|\left|q_{0}(x, y)\left\|\left.\right|_{p} \leqq\right\| q_{0}(x, y) \|\right|_{\sigma(p)} \leqq|(x, y)|_{p} \quad \text { and } \quad \inf _{x \in E}|(x, y)|_{p}\right. \\
=\inf _{x \in E}\left\|A_{p} y-x\right\|_{p}+\|\| y\left|\left\|\left.\right|_{\sigma(p)}=\right\|\left\|q_{0}(x, y)\right\| \|_{\sigma(p)} \cdot \quad \square\right.
\end{gathered}
$$

Let $E \hat{\times}_{\left(A_{p}\right)} F_{0}$ be the completion of the space $E \times_{\left(A_{p}\right)} F_{0}$. Using the above lemma and the next theorem we obtain that $E \hat{\times}_{\left(A_{p}\right)} F_{0}$ is a twisted sum of the spaces $E$ and $F$.

Theorem 2.4. Assume $E$ and $F$ are Fréchet spaces and $F_{0}$ is a dense subspace of $F$. If the sequence $0 \rightarrow E \stackrel{\dot{j}}{\rightarrow} X \stackrel{q_{0}}{\rightarrow} F_{0} \rightarrow 0$ is exact, then also the sequence $0 \rightarrow E \stackrel{\dot{j}}{\rightarrow}$ $\hat{X} \stackrel{q}{\rightarrow} F \rightarrow 0$ is exact. Here $\hat{X}$ is the completion of $X$ and $q$ is the continuous extension of $q_{0}$.

Proof. That the map $j: E \rightarrow \hat{X}$ is one to one is evident. Similarly $j(E) \subset \operatorname{ker} q$. Let $z \in \operatorname{ker} q$, and let $z_{n} \in X$ be a sequence converging to $z$. We have $\lim _{n \rightarrow \infty} q\left(z_{n}\right)=$ $q(z)=0$. Let $d$ be a translation invariant metric on $X$ compatible with the topology of $X$, and let $V_{n}=\{z \in X \mid d(z, 0)<1 / n\}$. Since $q_{0}: X \rightarrow F_{0}$ is an open mapping we may, by passing to a subsequence if necessary, assume that $q_{0}\left(z_{n}\right) \in q_{0}\left(V_{n}\right)$. Choose $\tilde{z}_{n} \in V_{n}$ such that $q_{0}\left(z_{n}\right)=q_{0}\left(\tilde{z}_{n}\right)$. We have $z_{n}-\tilde{z}_{n}=j\left(x_{n}\right)$ for some $x_{n} \in E$ and $\lim _{n \rightarrow \infty}\left(z_{n}-\tilde{z}_{n}\right)=z-0=z$. Since $E$ is complete, it follows that $z=j(x)$ for some $x \in E$. Therefore ker $q \subset j(E)$.

To show that $q: \hat{X} \rightarrow F$ is onto, let $y \in F=\bar{F}_{0}$. As before, there is a sequence $y_{n} \in F_{0}$ converging to $y$ with $\left(y-y_{n}\right)-\left(y-y_{n+1}\right) \in q_{0}\left(V_{2^{n}}\right)$. Let $z_{n} \in V_{2^{n}}$ be such that $q_{0}\left(z_{n}\right)=-y_{n}+y_{n+1}$. Since $\sum_{i=1}^{n} z_{i}, n \in N$, is a Cauchy sequence in $\hat{X}$, it converges. 
Let $z=\sum_{i=1}^{\infty} z_{i}$. We have $q(z)=\lim _{n \rightarrow \infty} \sum_{i=1}^{n} q\left(z_{i}\right)=\lim _{n \rightarrow \infty} \sum_{i=1}^{n}\left(y_{i+1}-y_{i}\right)=$ $\lim _{n \rightarrow \infty}\left(y_{n+1}-y_{1}\right)=y-y_{1}$. Hence $y=q(z)+y_{1}=q(\tilde{z})$ for some $\tilde{z} \in \hat{X}$.

Using the open mapping theorem we conclude that $j$ is an isomorphism onto its image and $q$ is an open mapping.

Assume now that $F$ has a basis. Let $F_{0}$ be the linear span of the basis vectors.

Theorem 2.5. Let $0 \rightarrow E \rightarrow X \rightarrow F \rightarrow 0$ be a short exact sequence. There exists a sequence of linear maps $A_{p}: F_{0} \rightarrow E$ such that

(i) there is a strictly increasing function $\sigma: N \rightarrow N$ such that for every $p>q$ we have

$$
\left\|A_{p} y-A_{q} y\right\|_{q} \leqq\|y\| \|_{\sigma(p)}, \quad y \in F_{0}, \quad \text { and }
$$

(ii) there is an isomoprhism $T: X \rightarrow E \hat{\times}_{\left(A_{p}\right)} F_{0}$ such that the following diagram commutes:

$$
\begin{gathered}
0 \underset{\mathrm{id}_{E} \downarrow}{\longrightarrow} E \underset{T}{\longrightarrow} X \underset{\mathrm{id}_{F} \downarrow}{\longrightarrow} F \rightarrow 0 \\
0 \longrightarrow E \longrightarrow \hat{\times}_{(A p)} F_{0} \longrightarrow F \rightarrow 0 .
\end{gathered}
$$

Proof. Let $\theta: F \rightarrow X$ be a linear map such that $q \theta=\mathrm{id}_{F}$, where $q$ is the quotient map. Let $j: E \rightarrow X$ be the embedding. Define $R: X \rightarrow E \times F$ by $R z=\left(j^{-1}(z-\theta q z)\right.$, $q(z))$. We get the following commutative diagram:

$$
\begin{aligned}
& \underset{\mathrm{id}_{E} \downarrow}{E} \underset{R \downarrow}{\stackrel{j}{\longrightarrow}} X \underset{\mathrm{id}_{\boldsymbol{F}} \downarrow}{\longrightarrow} F \rightarrow 0 \\
& 0 \longrightarrow E \stackrel{\tilde{j}}{\longrightarrow} E \times F \stackrel{\tilde{q}}{\rightarrow} F \rightarrow 0 \text {. }
\end{aligned}
$$

Here $\tilde{j}(x)=(x, 0)$ and $\tilde{q}(x, y)=y$. If $U \subset E \times F$ is defined to be open if and only if $R^{-1}(U)$ is open, it follows that we can identify $X$ with $E \times F$.

Let $\left(|(,)|_{p}\right)$ be an increasing sequence of seminorms on $E \times F$, which defines its topology. From the exactness of the sequence $0 \rightarrow E \rightarrow E \times F \rightarrow F \rightarrow 0$ it follows that there is an increasing function $\varrho: N \rightarrow N$ such that for every $p \in N$ we have

$$
\begin{gathered}
\|x\|_{p} \leqq|(x, 0)|_{\varrho(p)}, \\
\||y|\|_{p} \leqq|(x, y)|_{\varrho(p)}, \quad \text { and }
\end{gathered}
$$

(iii) for every basis vector $f_{m} \in F_{0}, m \in N$, there is a vector $x_{p, m} \in E$ such that $\left|\left(x_{p, m}, f_{m}\right)\right|_{p} \leqq \mid\left\|f_{m}\right\| \|_{\varrho(p)}$. Define $A_{p}: F_{0} \rightarrow E$ by $A_{p}\left(\sum_{m=1}^{\infty} a_{m}, f_{m}\right)=\sum_{m=1}^{\infty} a_{m} x_{\varrho(p), m}$. (Here $a_{m}=0$ except for finitely many $m \in N$.) Since the basis is absolute, we may assume that for every $p \in N||\left|\sum_{m=1}^{\infty} a_{m} f_{m}\right|\left\|_{p}=\sum_{m=1}^{\infty}\left|a_{m}\right|||\left|f_{m}\right|\right\|_{p}$. Therefore we have

$$
\left|\left(A_{p} y, y\right)\right|_{\varrho(p)} \leqq\left|\|y \mid\|_{\varrho(\varrho(p))}, \quad y \in F_{0} .\right.
$$


Fix $q$ and let $p>q$. From inequalities (i) and (iv), and from the triangle inequality we get

$$
\begin{gathered}
\left\|A_{p} y-A_{q} y\right\|_{q} \leqq\left|\left(A_{p} y-A_{q} y, 0\right)\right|_{\varrho(q)} \leqq\left(\left|\left(A_{p} y, y\right)\right|_{\varrho(p)}+\left|\left(A_{q} y, y\right)\right|_{\varrho(q)}\right) \\
\leqq 2 \mid\|y\|\left\|_{\varrho(\varrho(p))} \leqq\right\| y\|\|_{\sigma(p)},
\end{gathered}
$$

where $\sigma: N \rightarrow \boldsymbol{N}$ is a strictly increasing function.

We show next that the identity map id: $E \times F_{0} \rightarrow E \times_{\left(A_{p}\right)} F_{0}$ is continuous. This is a direct consequence of inequalities (i), (ii), and (iv):

$$
\begin{aligned}
& \left\|A_{p} y-x\right\|_{p}+\left|\left\|\left.y||\right|_{\sigma(p)} \leqq\left|\left(A_{p} y-x, 0\right)\right|_{\varrho(p)}+|\| y|||_{\sigma(p)}\right.\right. \\
& \leqq\left|\left\|\left.y\left|\|_{\varrho(\varrho(p))}+\right|(x, y)\right|_{\varrho(p)}+|(x, y)|_{\varrho(\sigma(p))} \leqq 3|(x, y)|_{r},\right.\right.
\end{aligned}
$$

where $r=\max \{\varrho(\varrho(\varrho(p))), \varrho(\sigma(p))\}$. Since $q: E \times F \rightarrow F$ is an open mapping, $E \times F_{0}$ is dense in $E \times F$. To conclude the proof, it is sufficient to show that the extension $\tilde{\text { id }}: E \times F \rightarrow E \hat{\times}_{\left(A_{p}\right)} F_{0}$ is an isomorphism. By the open mapping theorem we only need to show that it is bijective. For this we should note that the diagram

$$
\begin{aligned}
& \underset{ }{\longrightarrow \mathrm{id}_{E} \downarrow} \underset{\text { id }}{\longrightarrow} \underset{\downarrow}{\longrightarrow} \underset{\operatorname{id}_{F} \downarrow}{\longrightarrow} F \rightarrow 0 \\
& 0 \longrightarrow E \longrightarrow E \hat{\times}_{(A p)} F_{0} \rightarrow F 0
\end{aligned}
$$

is commutative and that its rows are exact. The exactness of the second row follows from Theorem 2.4 .

To construct nontrivial examples of short exact sequences $0 \rightarrow E \rightarrow X \rightarrow F \rightarrow 0$ we need to know whether or not they split, i.e., we need to know if $X$ can be identified in a canonical way with the product space $E \times F$. In terms of the linear maps $A_{p}$ we have the following criterion.

Theorem 2.6. A short exact sequence $0 \rightarrow E \rightarrow E \hat{\times}_{\left(A_{p}\right)} F_{0} \rightarrow F \rightarrow 0$ splits if and only if there is a linear map $A: F_{0} \rightarrow E$ and a function $\varrho: N \rightarrow \boldsymbol{N}$ such that

for all $y \in F_{0}$ and $p \in N$.

$$
\left\|A y-A_{p} y\right\|_{p} \leqq\|y\|_{\varrho(p)}
$$

Proof. If the sequence splits, there is a continuous linear map $T: F \rightarrow E \hat{\times}_{\left(A_{p}\right)} F$ such that $q T=\mathrm{id}_{F}$. Here, as before, $q$ is the extension of the quotient map $q_{0}(x, y)=y$, $(x, y) \in E \times F_{0}$. Fix $y \in F_{0}$. We have $q(T y-(0, y))=0$. Hence there is a unique element in $E$, call it $A y$, such that $T y-(0, y)=(A y, 0)$. Since $T$ is linear it follows that we get a linear map $A: F_{0} \rightarrow E$. The continuity of the map $T$ implies that there is a function $\varrho: N \rightarrow N$ such that for every $y \in F_{0}$ we have

$$
\left\|A y-A_{p} y\right\|_{p} \leqq\left\|A_{p} y-A y\right\|_{p}+\|\| y\left|\left\|_{\sigma(p)}=|(A y, y)|_{p}=|T y|_{p} \leqq\right\| y \|_{\varrho(p)} .\right.
$$

For the converse, let $T$ be the extension of the linear map $y \mapsto(A y, y)$. That it is continuous is a consequence of our assumption. Since $q(A y, y)=y, y \in F_{0}$, we have $q T y=y, y \in F$. 


\section{Examples}

In this section we shall apply the construction method just developed and give examples of non-splitting exact sequences $0 \rightarrow \Lambda_{1}(\alpha) \rightarrow X \rightarrow \Lambda_{1}(\beta) \rightarrow 0$ and $0 \rightarrow \Lambda_{\infty}(\alpha) \rightarrow$ $X \rightarrow \Lambda_{1}(\beta) \rightarrow 0$. Our method allows us to weaken the stability conditions present in the earlier constructions of Vogt [12].

Throughout this section $\alpha=\left(\alpha_{n}\right)$ and $\beta=\left(\beta_{m}\right)$ are exponent sequences, i.e., increasing sequences of positive numbers that go to infinity, and we shall assume that $\alpha$ is weakly stable, that is, $\sup _{n \in N} \alpha_{n+1} / \alpha_{n}<\infty$.

Lemma 3.1. Let $\left(t_{i}\right)$ be an increasing sequence of positive numbers with $\lim _{i \rightarrow \infty} t_{i}=\infty$ and denote

$$
I(m, i)=\left\{n \in \mathbf{N} \mid \beta_{m} / t_{i+1}<\alpha_{n} \leqq \beta_{m} / t_{i}\right\}
$$

for $m, i \in N$. Then there are arbitrarily large indices $i$, for which there are arbitrarily large $m$ with nonempty $I(m, i)$.

Proof. Let us assume on the contrary that there is $i_{0} \in N$ such that for every $i \geqq i_{0}$ there is $m_{i} \in N$ such that $I(m, i)=\emptyset$ if $m \geqq m_{i}$. Naturally we can select the sequence $\left(m_{i}\right)$ to be increasing. So for every $m \geqq m_{i_{0}}=m_{0}$ there is $j_{m} \geqq i_{0}$ such that $I(m, i)=\emptyset$ for every $i$ with $i_{0} \leqq i \leqq j_{m}$. This sequence $\left(j_{m}\right)$ can be chosen to be increasing and go to infinity. It then follows that the set $\left\{n \mid \beta_{m} / t_{j_{m}+1}<\alpha_{n} \leqq \beta_{m} / t_{i_{0}}\right\}$ is empty for $m \geqq m_{0}$; thus there are indices $n_{m}$ such that

$$
\alpha_{n_{m}} \leqq \beta_{m} / t_{j_{m}+1} \text { and } \beta_{m} / t_{i_{0}}<\alpha_{n_{m}+1} .
$$

Combining these inequalities we obtain

$$
\alpha_{n_{m}+1} / \alpha_{n_{m}} \geqq t_{j_{m}+1} / t_{i_{0}}
$$

for all $m \geqq m_{0}$. Since $\lim _{m \rightarrow \infty} t_{j_{m}+1}=\infty$, we have a contradiction with the weak stability of $\alpha$.

In Section 2 the systems of seminorms in $E$ and $F$ were chosen to be such that their unit balls form a neighbourhood basis of the origin. In the case of power series spaces it has become a custom to use the fundamental sequences of seminorms

and

$$
\|\xi\|_{p}=\sum_{n=1}^{\infty}\left|\xi_{n}\right| e^{p \beta_{n}}, \quad \xi=\left(\xi_{n}\right) \in \Lambda_{\infty}(\beta), \quad p \in N
$$

$$
\|\xi\|_{p}=\sum_{n=1}^{\infty}\left|\xi_{n}\right| e^{-(1 / p) \beta_{n}}, \quad \xi=\left(\xi_{n}\right) \in \Lambda_{1}(\beta), \quad p \in N .
$$

Multiplying these norms by sufficiently large positive scalars we get systems of seminorms of the kind used in Section 2. Therefore the compatibility condition has to be replaced by the following assertion: 
There is a function $\sigma: N \rightarrow N$ and positive numbers $C_{p}, p \in N$, such that

$$
\left\|A_{p} y-A_{q} y\right\|_{q} \leqq C_{p}\|y\| \|_{\sigma(p)} \text { for all } y \in F_{0} \text { and } p>q .
$$

The splitting condition will take the form:

There is a linear map $A: F_{0} \rightarrow E$, a function $\sigma: N \rightarrow N$ and positive numbers $C_{p}, p \in N$, such that $\left\|A y-A_{p} y\right\|_{p} \leqq C_{p}\|y\| \|_{\sigma(p)}$ for all $y \in F_{0}$ and $p \in N$.

Let $\left(e_{n}\right)$ and $\left(f_{m}\right)$ be the coordinate bases of $\Lambda_{1}(\alpha)$ and $\Lambda_{1}(\beta)$, respectively, and let $F_{0}$ be the linear span of the vectors $f_{m}, m \in N$. Denote

$$
I(m, i)=\left\{n \in N \mid \beta_{m} / i+1<\alpha_{n} \leqq \beta_{m} / i\right\}, \quad i \in N,
$$

and define the maps $A_{p}: F_{0} \rightarrow \Lambda_{1}(\alpha), p \in N$, by setting for every $m \in N$

$$
A_{p} f_{m}=\sum_{i=1}^{p} e^{(1 / i 2) \beta_{m}}(1 /|I(m, i)|) \sum_{n \in I(m, i)} e_{n},
$$

where $|I(m, i)|$ stands for the number of elements of the finite set $I(m, i)$. If $I(m, i)$ is empty, we put $(1 /|I(m, i)|) \sum_{n \in I(m, i)} e_{n}=0$.

Let $p>q$. Then

$$
\begin{gathered}
\left\|A_{p} f_{m}-A_{q} f_{m}\right\|_{q}=\sum_{i=q+1}^{p} e^{(1 / i 2) \beta_{m}}(1 /|I(m, i)|) \sum_{n \in I(m, i)}\left\|e_{n}\right\|_{q} \\
\leqq \\
\sum_{i=q+1}^{p} e^{(-1 / q(i+1)+1 / i 2) \beta_{m}} \leqq p e^{-(1 / \sigma(p)) \beta_{m}},
\end{gathered}
$$

where

$$
\sigma(p)=p^{3}(p+1) \geqq q i^{2}(i+1) /\left(i^{2}-q i-q\right),
$$

for $q<i \leqq p$. Thus the compatibility condition is satisfied and so the sequence of maps $\left(A_{p}\right)$ defines a twisted sum $X=\Lambda_{1}(\alpha) \hat{X}_{\left(A_{p}\right)} F_{0}$, which is a nuclear Fréchet space, if the spaces $\Lambda_{1}(\alpha)$ and $\Lambda_{1}(\beta)$ are nuclear. We shall show next that the short exact sequence $0 \rightarrow \Lambda_{1}(\alpha) \rightarrow X \rightarrow \Lambda_{1}(\beta) \rightarrow 0$ does not split. Assume the contrary; accordingly, there is a linear map $A: F_{0} \rightarrow \Lambda_{1}(\alpha), A f_{m}=\sum_{n=1}^{\infty} a_{m, n} e_{n}$, a function $\sigma: N \rightarrow N$ and positive numbers $C_{p}, p \in N$, such that

for all $m \in N$. Since

$$
\left\|A f_{m}-A_{p} f_{m}\right\|_{p} \leqq C_{p} e^{-(1 / \sigma(p)) \beta_{m}}
$$

$$
\sum_{i=1}^{p} \sum_{n \in I(m, i)}\left|a_{m, n}-e^{(1 / i) \beta_{m}} /\right| I(m, i)\left\|e^{-(1 / p) \alpha_{n}} \leqq\right\| A f_{m}-A_{p} f_{m} \|_{p}
$$

for a given $p$, we then have

$$
\sum_{n \in I(m, i)}\left|a_{m, n}-e^{(1 / i 2) \beta_{m}} /\right| I(m, i) \| e^{-(1 / i p) \beta_{m}} \leqq C_{p} e^{-(1 / \sigma(p)) \beta_{m}}
$$

for all $m \in N$ and $i=1,2, \ldots, p$ with $I(m, i) \neq \emptyset$. Consequently, for these indices $m$ and $i$ we have

$$
\sum_{n \in I(m, i)}\left|a_{m, n}\right| \geqq e^{(1 / i 2) \beta_{m}}-C_{p} e^{(1 / i p-1 / \sigma(p)) \beta_{m}}=e^{(1 / i 2) \beta_{m}}\left(1-C_{p} e^{\left.(1 / i p-1 / \sigma(p)-1 / i 2) \beta_{m}\right)} .\right.
$$


Choosing $p=i$ we obtain that for every $i \in N$ there is $\hat{m}_{i} \in N$ such that

$$
\sum_{n \in I(m, i)}\left|a_{m, n}\right| \geqq \frac{1}{2} e^{(1 / i 2) \beta_{m}}
$$

when $m \geqq \hat{m}_{i}$ and $I(m, i) \neq \emptyset$.

Let $p \in N$ be arbitrary. We then have, on the other hand, that

$$
\left\|A f_{m}-A_{p} f_{m}\right\|_{p} \geqq \sum_{i=p+1}^{\infty} \sum_{n \in I(m, i)}\left|a_{m, n}\right| e^{-(1 / p) \alpha_{n}} \geqq \sum_{i=p+1}^{\infty} e^{-(1 / i p) \beta_{m}} \sum_{n \in I(m, i)}\left|a_{m, n}\right| .
$$

According to Lemma 3.1. there exist arbitrarily large indices $i \geqq p+1$ for which we can be sure to find an index $m_{i} \geqq \hat{m}_{i}$ such that $I\left(m_{i}, i\right) \neq \emptyset$. For these $i$ we then obtain from the above inequalities

$$
C_{p} e^{-(1 / \sigma(p)) \beta_{m_{i}}} \geqq\left\|A f_{m_{i}}-A_{p} f_{m_{i}}\right\|_{p} \geqq \frac{1}{2} e^{(1 / i 2-1 / i p) \beta_{m_{i}}}=\frac{1}{2} e^{-(i-p) /(i 2 p) \beta_{m_{i}}} .
$$

Since $\lim _{i \rightarrow \infty}(i-p) / i^{2} p=0$, we have a contradiction.

Although the short exact sequence $0 \rightarrow \Lambda_{1}(\alpha) \rightarrow X \rightarrow \Lambda_{1}(\beta) \rightarrow 0$ constructed above does not split, the twisted sum $X=\Lambda_{1}(\alpha) \hat{X}_{\left(A_{p}\right)} F_{0}$ is, at least in the stable case, isomorphic to the product space $\Lambda_{1}(\alpha) \times \Lambda_{1}(\beta)$ [12]. On the other hand, nothing is known about the nontrivial twisted sum of the spaces $\Lambda_{\infty}(\alpha)$ and $\Lambda_{1}(\beta)$ that will be constructed next. The construction is very similar to the previous one.

In this case we define for $m, i \in \boldsymbol{N}$

$$
I(m, i)=\left\{n \in N \mid \beta_{m} /(i+1)^{2}<\alpha_{n} \leqq \beta_{m} / i^{2}\right\} .
$$

Let $F_{0}$ be the linear span of the coordinate basis vectors $f_{m}$ of $\Lambda_{1}(\beta)$, and let $\left(e_{n}\right)$ be the coordinate basis of $\Lambda_{\infty}(\alpha)$. For every $p \in N$ we define a map $A_{p}: F_{0} \rightarrow \Lambda_{\infty}(\alpha)$,

$$
A_{p} f_{m}=\sum_{i=1}^{p} e^{-(1 / i+1) \beta_{m}}(1 /|I(m, i)|) \sum_{n \in I(m, i)} e_{n}, \quad m \in \mathbf{N} .
$$

For $p>q$ we now have

where

$$
\begin{gathered}
\left\|A_{p} f_{m}-A_{q} f_{m}\right\|_{q}=\sum_{i=q+1}^{p} e^{-(1 / i+1) \beta_{m}}(1 /|I(m, i)|) \sum_{n \in I(m, i)} e^{q x_{n}} \\
\leqq \sum_{i=q+1}^{p} e^{\left(q / i^{2}-1 /(i+1)\right) \beta_{m}} \leqq p e^{-(1 / \sigma(p)) \beta_{m}},
\end{gathered}
$$

$$
\sigma(p)=p^{2}(p+1) \geqq i^{2}(i+1) /\left(i^{2}-i q-q\right),
$$

for $q<i \leqq p$. Thus the sequence of maps $\left(A_{p}\right)$ defines a twisted sum $X$ of $\Lambda_{\infty}(\alpha)$ and $\Lambda_{1}(\beta), X=\Lambda_{\infty}(\alpha) \hat{X}_{\left(A_{p}\right)} F_{0}$. To prove that this twisted sum is not trivial, assume that there is a linear map $A: F_{0} \rightarrow \Lambda_{\infty}(\alpha)$, a function $\sigma: N \rightarrow N$ and positive numbers $C_{p}, p \in N$, such that the following is true for every $m \in N$

$$
\left\|A f_{m}-A_{p} f_{m}\right\|_{p} \leqq C_{p} e^{-(1 / \sigma(p)) \beta_{m}} .
$$


Let

then

$$
A f_{m}=\sum_{n=1}^{\infty} a_{m, n} e_{n}
$$

$$
\sum_{n \in I(m, i)} \mid a_{m, n}-e^{-(1 /(i+1)) \beta_{m}} / I(m, i) \| e^{\left(p /(i+1)^{2}\right) \beta_{m}} \leqq C_{p} e^{-(1 / \sigma(p)) \beta_{m}}
$$

for all $p \in N$ and for all $m$ and $i=1, \ldots, p$ with nonempty $I(m, i)$. Choosing $p=i+1$ we obtain

$$
\begin{gathered}
\sum_{n \in I(m, i)}\left|a_{m, n}\right| \geqq e^{-(1 /(i+1)) \beta_{m}}-C_{i+1} e^{(-1 / \sigma(i+1)-1 /(i+1)) \beta_{m}} \\
=e^{-(1 /(i+1)) \beta_{m}}\left(1-C_{i+1} e^{\left.-(1 / \sigma(i+1)) \beta_{m}\right)} .\right.
\end{gathered}
$$

Thus for every $i$ there is an index $\hat{m}_{i}$ such that

$$
\sum_{n \in I(m, i)}\left|a_{m, n}\right| \geqq \frac{1}{2} e^{-(1 /(i+1)) \beta_{m}}
$$

when $m \geqq \hat{m}_{i}$ and $I(m, i) \neq \emptyset$.

Let $p \in N$ be arbitrary. Then

$$
\left\|A f_{m}-A_{p} f_{m}\right\|_{p} \geqq \sum_{i=p+1}^{\infty} \sum_{n \in I(m, i)}\left|a_{m, n}\right| e^{p x_{n}} \geqq \sum_{i=p+1}^{\infty} e^{\left(p /(i+1)^{2}\right) \beta_{m}} \sum_{n \in I(m, i)}\left|a_{m, n}\right| .
$$

According to Lemma 3.1 we can choose arbitrarily large indices $i$ and $m_{i} \geqq \hat{m}_{i}$ such that $I\left(m_{i}, i\right) \neq \emptyset$. Combining the derived inequalities we then obtain for these $i$

$$
C_{p} e^{-(1 / \sigma(p)) \beta_{m_{i}}} \geqq\left\|A f_{m_{i}}-A_{p} f_{m_{i}}\right\|_{p} \geqq \frac{1}{2} e^{-\left(i /(i+1)^{2) \beta_{m_{i}}}\right.},
$$

where we have a contradiction.

The twisted sum of $\Lambda_{\infty}(\alpha)$ and $\Lambda_{1}(\beta)$ constructed above remains unexplored. The general question is what kinds of nuclear Fréchet spaces one gets by forming twisted sums of nuclear Fréchet spaces with bases. As one would guess, not every nuclear Fréchet space can be expressed in this way. Let $X=E \hat{\times}_{\left(A_{p}\right)} F_{0}$ be a twisted sum of nuclear Fréchet spaces $E$ and $F$ and suppose that $X$ admits a continuous norm. It is easily seen that the canonical map between the completions of the spaces $\left(X,|(,)|_{p+1}\right)$ and $\left(X,|(,)|_{p}\right)$ is one to one, if the corresponding maps of $E$ and $F$ are one to one. It follows that a twisted sum of nuclear spaces with bases is countably normed. However, Dubinsky [5] has constructed a nuclear Fréchet space with continuous norm which is not countably normed. 


\section{Splitting an exact sequence}

In his theory on characterizations of subspaces and quotients of certain nuclear Fréchet spaces Vogt has introduced the properties DN and $\Omega$ and has shown that if $E$ has $\Omega$ and $F$ has DN, then every short exact sequence $0 \rightarrow E \rightarrow X \rightarrow F \rightarrow 0$ splits [11], [13]. Generalizations of these ideas have been presented in [1], [2] and in the talk given by Vogt in the Colloquium on Nuclear Spaces in Ankara, June 1981. For example, a condition on $E$ and $F$ has been given (see (V) below), which quarantees the splitting of sequences $0 \rightarrow E \rightarrow X \rightarrow F \rightarrow 0$. In what follows, we shall require that both spaces have bases. Then we can give a weaker splitting condition which is also almost a necessary condition for a short exact sequence to split.

Theorem 4.1. Assume $E$ and $F$ are nuclear Fréchet spaces with bases. Then every short exact sequence $0 \rightarrow E \rightarrow X \rightarrow F \rightarrow 0$ splits if for every function $\sigma: N \rightarrow N$ there is a function $\varrho: N \rightarrow N$ such that for every $p>q$ we have

$$
\sum_{r=q}^{p-1}\left\|x^{\prime}\right\|_{r}\left|\|y\|\left\|_{\sigma(r)} \leqq\right\| x^{\prime}\left\|_{q}\right\| y\right|\left\|_{\varrho(q)}+\right\| x^{\prime}\left\|_ { p } \left|\|y \mid\|_{\varrho(p)}\right.\right.
$$

where $x^{\prime} \in E^{\prime}$ and $y \in F$. Here $\left\|x^{\prime}\right\|_{r}=\sup \left\{\mid\left\langle x, x^{\prime}\right\rangle\|\| x \|_{r} \leqq 1\right\}$.

Proof. Let $\left(e_{n}\right)$ be a basis of $E$ and let $\left(e_{n}^{\prime}\right)$ be the corresponding sequence of biorthogonal functionals. For the space $F$ we have similarly a basis $\left(f_{m}\right)$ and biorthogonal functionals $\left(f_{m}^{\prime}\right)$. By nuclearity we may assume that the corresponding seminorms satisfy the equations

and

$$
\left\|\sum_{n=1}^{\infty} a_{n} e_{n}\right\|_{p}=\max _{n}\left|a_{n}\right|\left\|e_{n}\right\|_{p}
$$

$$
\left\|\sum_{m=1}^{\infty} b_{m} f_{m}\right\|\left\|_{p}=\sum_{m=1}^{\infty}\left|b_{m}\right| \quad\right\||| f_{m}\|\|_{p} .
$$

In particular, the series $\sum_{n=1}^{\infty} a_{n} e_{n}$ converges if $\sup _{n}\left|a_{n}\right|\left\|e_{n} \mid\right\|_{p}<\infty$ for every $p$.

By Theorem 2.5 there are linear maps $A_{p}$ from the linear span $F_{0}$ of the basis vectors $\left(f_{m}\right)$ to $E$ such that $X$ can be identified with $E \hat{\times}_{\left(A_{p}\right)} F_{0}$. Also there is a function $\sigma: N \rightarrow N$ such that for every $p$ we have

$$
\left\|A_{p+1} y-A_{p} y\right\|_{p} \leqq\|y\|_{\sigma(p)}, \quad y \in F_{0} .
$$

Let $\varrho: N \rightarrow N$ be the function given by our assumption. According to Theorem 2.6 it is sufficient to construct a linear map $A: F_{0} \rightarrow E$ such that for every $p$ we have

$$
\left\|A y-A_{p} y\right\|_{p} \leqq\|y \mid\|_{\varrho(p)}, \quad y \in F_{0} .
$$


We do this coordinatewise. Fix $n$ and $m$ and let for the moment $p>q$. By our assumption we have

$$
\begin{aligned}
\left\langle A_{p} f_{m}, e_{n}^{\prime}\right\rangle-\left\langle A_{q} f_{m}, e_{n}^{\prime}\right\rangle & \leqq \sum_{r=q}^{p-1}\left|\left\langle A_{r+1} f_{m}, e_{n}^{\prime}\right\rangle-\left\langle A_{r} f_{m}, e_{n}^{\prime}\right\rangle\right| \\
\leqq & \sum_{r=q}^{p-1}\left\|e_{n}^{\prime}\right\|_{r}\left\|f_{m}\right\|\left\|_{\sigma(r)} \leqq\right\| e_{n}^{\prime}\left\|_{q}\right\| f_{m}\|\|_{Q(q)}+\left\|e_{n}^{\prime}\right\|_{p}\left\|f_{m}\right\| \|_{Q(p)} .
\end{aligned}
$$

Therefore

$$
\left\langle A_{p} f_{m}, e_{n}^{\prime}\right\rangle-\left\|e_{n}^{\prime}\right\|_{p}\left\|f_{m}\right\|\left\|_{\varrho(p)} \leqq\left\langle A_{q} f_{m}, e_{n}^{\prime}\right\rangle+\right\| e_{n}^{\prime}\left\|_{q}\right\|\left\|f_{m}\right\| \|_{\varrho(p)} .
$$

Since this is true for every $p$ and $q$, there is a real number $x(n, m)$ for which we have

and

$$
x(n, m) \leqq \inf _{q}\left\{\left\langle A_{q} f_{m}, e_{n}^{\prime}\right\rangle+\left\|e_{n}^{\prime}\right\|_{q}\left\|f_{m}\right\|_{\varrho(q)}\right\}
$$

$$
x(n, m) \geqq \sup _{p}\left\{\left\langle A_{p} f_{m}, e_{n}^{\prime}\right\rangle-\left\|e_{n}^{\prime}\right\|_{p}\left\|f_{m}\right\|_{\ell(p)}\right\} .
$$

Hence for every $p$ we have

$$
\left|x(n, m)-\left\langle A_{p} f_{m}, e_{n}^{\prime}\right\rangle\right| \leqq\left\|e_{n}^{\prime}\right\|_{p} \mid\left\|f_{m}\right\| \|_{\varrho(p)} .
$$

It follows that the series $\sum_{n=1}^{\infty} x(n, m) e_{n}$ converges, and that we have for every $p$

$$
\begin{gathered}
\left\|\sum_{n=1}^{\infty} x(n, m) e_{n}-A_{p} f_{m}\right\|_{p}=\left\|\sum_{n=1}^{\infty}\left(x(n, m)-\left\langle A_{p} f_{m}, e_{n}^{\prime}\right\rangle\right) e_{n}\right\|_{p} \\
=\max _{n} \mid x(n, m)-\left\langle A_{p} f_{m}, e_{n}^{\prime}\right\rangle\left\|e_{n}\right\|_{p} \leqq\left\|f_{m}\right\| \|_{\varrho(p)} .
\end{gathered}
$$

Therefore, if we define $A: F_{0} \rightarrow E$ to be the linear extension of the correspondence $f_{m} \mapsto \sum_{n=1}^{\infty} x(n, m) e_{n}$, we get a linear map which has the required property.

Assume now that every short exact sequence $0 \rightarrow E \rightarrow X \rightarrow F \rightarrow 0$ splits. Let $\sigma: N \rightarrow N$ be a function and let $N_{m}, m \in N$, be any sequence of finite subsets of $N$. Define linear maps $A_{p}: F_{0} \rightarrow E$ inductively by $A_{1}=0$ and

$$
A_{p+1} f_{m}=A_{p} f_{m}+\mid\left\|f_{m}\right\|_{\sigma(p)} \sum_{n \in N_{m}}\left\|e_{n}^{\prime}\right\|_{p} e_{n} .
$$

It follows from Theorem 2.4 that we have a short exact sequence $0 \rightarrow E \rightarrow E \hat{\times}_{\left(A_{p}\right)} F_{0} \rightarrow$ $F \rightarrow 0$. Since by our assumption it splits, there is, by Theorem 2.6, a linear map $A: F_{0} \rightarrow E$ and a function $\varrho: N \rightarrow N$ for which we have

$$
\left\|A y-A_{p} y\right\|_{p} \equiv\|y\| \|_{\varrho(p)}
$$

for every $p$. Using maximum seminorms in $E$ we get

$$
\left|\left\langle A f_{m}, e_{n}^{\prime}\right\rangle-\left\langle A_{p} f_{m}, e_{n}^{\prime}\right\rangle\right| \leqq\left\|e_{n}^{\prime}\right\|_{p}\left\|f_{m}\right\|_{\varrho(p)}
$$


for every $p$. Therefore for $p>q$ and $n \in N_{m}$ we have

$$
\begin{gathered}
\sum_{r=q}^{p-1}\left|\left\|f_{m}\right\|\left\|_{\sigma(r)}\right\| e_{n}^{\prime} \|_{r}=\right| \sum_{r=q}^{p-1}\left(\left\langle A_{r+1} f_{m}, e_{n}^{\prime}\right\rangle-\left\langle A_{r} f_{m}, e_{n}^{\prime}\right\rangle\right) \mid \\
\equiv\left|\left\langle A_{g} f_{m}, e_{n}^{\prime}\right\rangle-\left\langle A f_{m}, e_{n}^{\prime}\right\rangle\right|+\left|\left\langle A f_{m}, e_{n}^{\prime}\right\rangle-\left\langle A_{p} f_{m}, e_{n}^{\prime}\right\rangle\right| \leqq\left\|e_{n}^{\prime}\right\|_{q}\left|\| f _ { m } \| \| _ { \varrho ( q ) } + \| e _ { n } ^ { \prime } \left\|_ { p } \left|\left\|f_{m} \mid\right\|_{Q(p)},\right.\right.\right.
\end{gathered}
$$

i.e., the converse of our theorem holds with the restriction $n \in N_{m}$.

One of the splitting conditions in use is the following (cf. [1, III. 2] and [2]): $\exists p_{0} \forall q_{0} \exists q \forall p \forall r \exists s$

(V)

$$
\left\|x^{\prime}\right\|_{q}\|y\|_{p} \leqq\left\|x^{\prime}\right\|_{q_{0}} \mid\|y\|_{p_{0}}+\left\|x^{\prime}\right\|_{r}\|y\| \|_{s},
$$

where $x^{\prime} \in E^{\prime}$ and $y \in F$. We are going to show that this condition implies the one we have in Theorem 4.1. We need the following lemma used in the context of splitting theorems [1, III. 2].

Lemma 4.2. Assume E and $F$ satisfy the above mentioned condition (V), and let $\sigma: N \rightarrow N$ be a function. Then there is a natural number $p$, a sequence $\left(r_{i}\right)$ of natural numbers, and a function $\varrho: N \rightarrow N$ such that for every $i \quad \varrho\left(r_{i}\right) \geqq \max \left\{\sigma\left(r_{i}\right), p_{0}\right\}$ and

$$
\left\|x^{\prime}\right\|_{r_{i}}\|y\|\left\|_{Q\left(r_{i}\right)} \leqq 2^{-r_{i}}\right\| x^{\prime}\left\|_{r_{i-1}}\right\| y\left\|_{p}+\right\| x^{\prime}\left\|_{r_{i+1}}\right\| y\|\|_{Q\left(r_{i+1}\right)},
$$

where $x^{\prime} \in E^{\prime}$ and $y \in F$.

Proof. We give only the induction step of the proof. Let $p_{0}$ be the natural number given by $(V)$. Assume we have chosen $r_{j}$ and defined $\varrho\left(r_{j}\right)$ such that our claim holds for $j \cong i$ and that $\forall p \forall r \exists s$

$$
\left\|x^{\prime}\right\|_{r_{i+1}}\|y\|\left\|_{p} \leqq\right\| x^{\prime}\left\|_{r_{i}}\right\| y\left\|_{p_{0}}+\right\| x^{\prime}\left\|_{r}\right\| y \|_{s} .
$$

Using (V), choose $r_{i+2}$ such that $\forall p^{\prime} \forall r^{\prime} \exists s^{\prime}$

$$
\left\|x^{\prime}\right\|_{r_{i+2}}\left|\left\|y \left|\left\|_{p^{\prime}} \leqq\right\| x^{\prime}\left\|_{r_{i+1}}\right\| y\|\|_{p_{0}}+\left\|x^{\prime}\right\|_{r^{\prime}}\|y \mid\|_{s^{\prime}} .\right.\right.\right.
$$

Choose $p$ such that $2^{r_{i+1}}\|y\|_{o\left(r_{i+1}\right)} \equiv\|y\|_{p}$, and let $r=r_{i+2}$. For some $s$ we have

$$
\left\|x^{\prime}\right\| r_{r_{i+1}}\|y\|_{Q\left(r_{i+1}\right)} \leqq 2^{-r_{i+1}}\left\|x^{\prime}\right\|_{r_{i+1}}\|y\|\left\|_{p} \leqq 2^{-r_{i+1}}\right\| x^{\prime}\left\|_{r_{i}}\right\| y\left\|_{p_{0}}+\right\| x^{\prime}\left\|_{r_{i+2}}\right\| y\|\|_{s} .
$$

Let $\varrho\left(r_{i+2}\right)$ be the maximum of this $s$ and the number $\max \left\{\sigma\left(r_{i+2}\right), p_{0}\right\}$.

Corollary 4.3 Let $E$ and $F$ be nuclear Fréchet spaces with bases. Assume that $E$ and $F$ satisfy the condition (V). Then every short exact sequence $0 \rightarrow E \rightarrow X \rightarrow F \rightarrow 0$ splits.

Proof. Let $\sigma: N \rightarrow N$ be a function. According to Lemma 4.2 there is a function $\varrho: N \rightarrow N$ such that, passing to a subsequence of seminorms, we have for every $r$

$$
\left\|x^{\prime}\right\|_{r}\|y\|_{\sigma(r)} \leqq\left\|x^{\prime}\right\|_{r}\|y\|\left\|_{\varrho(r)} \leqq 2^{-r}\right\| x^{\prime}\left\|_{r-1}\right\| y\left\|_{p_{0}}+\right\| x^{\prime}\left\|_{r+1}\right\| y \|_{\varrho(r+1)} .
$$

Let $p>r>q$. We have

$$
\begin{aligned}
& \left\|x^{\prime}\right\|_{r}\|y\|\left\|_{\sigma(r)} \leqq 2^{-r}\right\| x^{\prime}\left\|_{q}\right\| y\|\|_{p_{0}}+\left\|x^{\prime}\right\|_{r+1}\|y y\| \|_{Q(r+1)} \\
& \leqq 2^{-r}\left\|x^{\prime}\right\|_{q}\|y\|\left\|_{p_{0}}+2^{-(r+1)}\right\| x^{\prime}\left\|_{q}\right\| y\|\|_{p_{9}}+\left\|x^{\prime}\right\|_{r+2}\|y\| \|_{Q(r+2)} \\
& \equiv \ldots \leqq 2^{-r+1}\left\|x^{\prime}\right\|_{q}\|y\|\left\|_{p_{0}}+\right\| x^{\prime}\left\|_{p}\right\| y\|\|_{Q(p)} .
\end{aligned}
$$


Hence

$$
\begin{aligned}
\sum_{r=q}^{p-1}\left\|x^{\prime}\right\|_{r}\|y\|_{\sigma(r)} & \leqq\left\|x^{\prime}\right\|_{q}\|y\|\left\|_{\sigma(q)}+2^{-q+1}\right\| x^{\prime}\left\|_{q}\right\| y\left\|_{p_{0}}+p\right\| x^{\prime}\left\|_{p}\right\| y\|\|_{\varrho(p)} \\
& \leqq 2\left\|x^{\prime}\right\|_{q}\|y \mid\|\left\|_{\varrho(q)}+p\right\| x^{\prime}\left\|_{p}\right\| y\|\|_{\varrho(p)} .
\end{aligned}
$$

If the values of the function $\varrho$ are increased appropriately, it follows that the assumption of Theorem 4.1 is satisfied for a subsequence of seminorms. Since this subsequence generates the original topology of $E$, the proof is complete.

In his proof of the above result Vogt used a Mittag--Leffier type procedure to construct a continuous linear converse of the quotient map. Our construction in Theorem 4.1 is, instead, based on an argument analogous to one used in the proof of Hahn-Banach theorem.

\section{References}

[1] Ahonen, H.: On nuclear Köthe spaces defined by Dragilev functions. - Ann. Acad. Sci. Fenn. Ser. A I Math. Dissertationes 38, 1981, 1-57.

[2] Aprola, H.: Characterization of subspaces and quotients of nuclear $L_{f}(\alpha, \infty)$-spaces. Compositio Math. (to appear).

[3] Dierolf, S.: Über Vererbbarkeitseigenschaften in topologischen Vektorräumen. - Thesis, Ludwig-Maximilians-Universität, Munich, 1974.

[4] Dubinsky, E.: The structure of nuclear Fréchet spaces. - Lecture Notes in Mathematics 720, Springer-Verlag, Berlin-Heidelberg-New York, 1979.

[5] Dubinsky, E.: Nuclear Fréchet spaces without the bounded approximation property. Studia Math. 71: 1, 1981, 85-105.

[6] Dynin, A. S., and B. S. Mitjagin: Criterion for nuclearity in terms of approximative dimension. -Bull. Acad. Polon. Sér. Sci. Math. Astronom. Phys. 8: 8, 1960, 535-540.

[7] Kalton, N. J.: The three space problem for locally bounded F-spaces. - Compositio Math. 37: 3, 1978, 243-276.

[8] Kalton, N. J.: Convexity, type and the three space problem. - Studia Math. 69: 3, 1981, $247-287$.

[9] Kalton, N. J., and N. T. Peck: Twisted sums of sequence spaces and the three space problem. - Trans. Amer. Math. Soc. 255, 1979, 1-30.

[10] Pietsch. A.: Nuclear locally convex spaces. - Springer-Verlag, Berlin--Heidelberg--New York, 1972.

[11] Vogt, D.: Characterisierung der Unterräume von s. - Math. Z. 155, 1977, 109-117.

[12] Vogt, D.: Eine Characterisierung der Potenzreihenräume von endlichem Typ und ihre Folgerungen. - Preprint.

[13] VogT, D., and M. J. WAGNer: Charakterisierung der Unterräume und Quotienträume der nuklearen stabilen Potenzreihenräumen von unendlichem Typ. - Studia Math. 70:1, 1981, 63-80.

University of Helsinki

Department of Mathematics

SF-00100 Helsinki 10

Finland

Received 20 May 1982 